\title{
Study on bubble-induced turbulence in pipes and containers with Reynolds-stress models
}

\author{
Yixiang Liao' ${ }^{1}$ Tian $M^{1,2}(\varangle)$ \\ 1. Institute of Fluid Dynamics, Helmholtz-Zentrum Dresden-Rossendorf, Bautzner Landstraße 400, 01328 Dresden, Germany \\ 2. Department of Civil and Environmental Engineering, Duke University, Durham, NC 27708, USA
}

\begin{abstract}
Bubbly flow still represents a challenge for large-scale numerical simulation. Among many others, the understanding and modelling of bubble-induced turbulence (BIT) are far from being satisfactory even though continuous efforts have been made. In particular, the buoyancy of the bubbles generally introduces turbulence anisotropy in the flow, which cannot be captured by the standard eddy viscosity models with specific source terms representing BIT. Recently, on the basis of bubble-resolving direct numerical simulation data, a new Reynolds-stress model considering BIT was developed by Ma et al. (J Fluid Mech, 883: A9 (2020)) within the Euler-Euler framework. The objective of the present work is to assess this model and compare its performance with other standard Reynolds-stress models using a systematic test strategy. We select the experimental data in the BIT-dominated range and find that the new model leads to major improvements in the prediction of full Reynolds-stress components.

Keywords

bubble-induced turbulence (BIT)

Reynolds-stress turbulence model

pipe flow

Euler-Euler two-fluid model

\section{Article History}

Received: 20 July 2021

Revised: 19 October 2021

Accepted: 1 November 2021

Research Article

(c) The Author(s) 2021
\end{abstract}

\section{Introduction}

Bubble-laden turbulent flows occur in a large number of processes in the energy generation, chemical industry, and nature (Lohse, 2018). In large-scale CFD simulations of these flows, the Euler-Euler (EE) method coupled with Reynolds-Averaged Navier-Stokes (RANS) modelling is the only viable approach. In this EE-RANS method, only continuous statistical quantities are calculated, and hence, all two-phase phenomena related to the gas-liquid phase boundaries need to be modelled (Ishii and Hibiki, 2006). In particular, one of the most challenging and complicated topics is turbulence modelling (Balachandar and Eaton, 2010) since the bubbles can strongly alter the carrier phase turbulence. One can readily cite several related mechanisms: (i) turbulence production due to the bubble wakes (Riboux et al., 2010); (ii) enhanced turbulence dissipation rate in the vicinity of the bubble surface (Ilić, 2006; Santarelli et al., 2016); (iii) modulation of the liquid mean velocity profile due to interphase momentum transfer, resulting in a change of shear-induced turbulence (SIT) (Lu and Tryggvason, 2013; du Cluzeau et al., 2019; Bragg et al., 2021); (iv) increased turbulence intermittency in the dissipation range (Ma et al.,
2021). These mechanisms can significantly affect the interfacial processes, such as bubble coalescence and breakup (Liao et al., 2014; Liao, 2020; Qi et al., 2020) and interphase momentum transfer (Lucas et al., 2016; Michaelides et al., 2016; Sommerfeld, 2017). Hence, providing an improved representation of some of these mechanisms is the goal of the turbulence modelling development for bubbly flows (Wörner and Erdogan, 2013; Lucas et al., 2015).

Over the last three decades, remarkable works have been accomplished in developing two-equation RANS models with source terms capturing the so-called bubble-induced turbulence (BIT) (Lopez de Bertodano et al., 1994; Morel, 1997; Troshko and Hassan, 2001; Rzehak and Krepper, 2013; Colombo and Fairweather, 2015; Ma, 2017; Vaidheeswaran and Hibiki, 2017; Liu et al., 2018; Liao et al., 2019; Magolan et al., 2019). These linear eddy-viscosity models take the bubble influence into account by adding source terms in the turbulence transport equations for both the turbulent kinetic energy (TKE, $k$ ) and a turbulence length-scale related parameter, e.g., the turbulence dissipation rate $\varepsilon$. As a result, this modulate as well the eddy-viscosity, by taking the expression $v_{\mathrm{t}}=C_{\mu} k^{2} / \varepsilon$ for a $k-\varepsilon$ type model for instance $\left(C_{\mu}=0.09\right.$, typically). However, despite the fact that these

\footnotetext{
$\triangle$ tian.ma@hzdr.de
} 
two-equation RANS models may be able to compute $k$ and $\varepsilon$ sufficiently, this type of model suffers from some issues concerning the standard eddy-viscosity concept:

$$
-\overline{u_{i}^{\prime} u_{j}^{\prime}}=\underbrace{C_{\mu} \frac{k^{2}}{\varepsilon}}_{v_{\mathrm{t}}}\left(\frac{\partial \bar{u}_{i}}{\partial x_{j}}+\frac{\partial \bar{u}_{j}}{\partial x_{i}}\right)-\frac{2}{3} \delta_{i j} k
$$

when applied to bubble-laden turbulent flows, as discussed in Ma et al. (2020a, 2020b). Here, $\delta_{i j}$ is the Kronecker delta, $u^{\prime} \equiv u-\bar{u}$ is the fluctuating liquid velocity, and $\cdots$ denotes a Reynolds average (estimated using appropriate space and time average).

For most single-phase flows, the turbulence production highly relates to the mean velocity gradients doing work on the Reynolds stresses, $\overline{u_{i}^{\prime} u_{j}^{\prime}}$, in the fluid, so that there is some rational for the eddy-viscosity concept. In BIT-dominated flows, however, the turbulence production mainly depends upon the interfacial energy transfer between liquid and bubbles (Kataoka and Serizawa, 1989). As a result, closures for $\overline{u_{i}^{\prime} u_{j}^{\prime}}$ based on the mean velocity gradient may not be meaningful for such a flow-at the basic level, the deficiency remains for the representation of $\overline{u_{i}^{\prime} u_{j}^{\prime}}$ in Eq. (1) that is intrinsic to this general hierarchy of EE $k-\varepsilon$ type model, hence, cannot be overcome by more complex BIT terms in the corresponding turbulence transport equations.

In view of these issues, several groups have sought to develop differential Reynolds-stress models (RSMs) based on the EE method that are more appropriate for BIT-dominated flows. We try to category their different Reynolds-stress closures into two classes. The first class refers to the works simply adapting the single-phase RSM in EE simulations of bubble-laden flows without considering the BIT effect. One of those examples can be taken in the study of Masood et al. (2014), who applied different single-phase RSMs in EE simulations of a bubble plume of Deen et al. (2001) and showed good agreement in the liquid single-point statistics with the experimental data. However, the success in this special case cannot be generalized to all kinds of bubbly flows. In contrast, the experiment of Deen et al. (2001) has also been simulated very accurately in terms of averaged liquid statistics by using different kinds of scale-resolving simulations without considering any BIT effect (Deen et al., 2001; Ničeno et al., 2008; Ma et al., 2015a; Ullrich et al., 2021). In these cited references, they show that compared to BIT the undulatory modulation of bubble plume is the dominant effect in such a flow, generating the most velocity fluctuations. Hence, considering the BIT effect or not influences the simulation only to a relatively small extent.

The BIT effect is considered in the second class of RSM applied to bubbly flows. Early attempts in this category, e.g., Lopez de Bertodano et al. (1990) combined the RSM of Launder et al. (1975) (LRR for short) with the BIT expression by Biesheuvel and van Wijngaarden (1984) as a source tensor to capture the turbulence generated by bubbles. This algebraic BIT expression is derived by using the assumption of a potential flow and considers the liquid velocity fluctuations influenced by the moving bubbles primarily from the displacement of the liquid. However, such an assumption cannot be applied for real situations of finite-size bubbles (say several millimetres), where the wake effect is dominant (Riboux et al., 2010). Further EE-RSMs for bubbly flows were performed by Cokljat et al. (2006), Colombo and Fairweather (2015), and recently by Ullrich et al. (2021). While Cokljat et al. (2006) and Ullrich et al. (2021) employed an isotropic source tensor to capture BIT, Colombo and Fairweather (2015) used a larger BIT source term in the direction of slip velocity between bubble and fluid. In all the above studies, the additional BIT tensor in the RSM resulted from various phenomenological modelling arguments.

Recently, Ma et al. (2020b) developed an RSM based on a term-by-term modelling strategy, having the interface resolving direct numerical simulation (DNS) data (Santarelli and Fröhlich, 2015, 2016) as a reference for the particular term in the modelled Reynolds-stress transport equation. The focus of the present work is on the application of this model in different BIT dominant flows to assess its prediction on all the Reynolds-stress components. After introducing some technical details of this RSM in Section 2, a brief summary of the validation cases and the simulation setup are provided in Section 3. The results obtained by the RSM and its comparison with the experimental data are described in Section 4. A conclusion on the model performance is given in Section 5 .

\section{Reynolds-stress model with BIT source tensor}

In this section, the complete formulation of the Reynoldsstress model of Ma et al. (2020b) is presented. First, the transport equations for the Reynolds stresses are given:

$$
\begin{aligned}
& \frac{\mathrm{D}\left(\alpha_{1} \overline{u_{i}^{\prime} u_{j}^{\prime}}\right)}{\mathrm{D} t}=-\underbrace{\underbrace{\frac{\partial}{\partial x_{k}}\left[\alpha_{1}\left(v_{1}+c_{\mathrm{s}} v_{\mathrm{t}}\right) \frac{\partial \overline{u_{i}^{\prime} u_{j}^{\prime}}}{\partial x_{\mathrm{k}}}\right]}_{D_{i j}: \text { diffusion }}-\underbrace{\alpha_{1} \frac{2}{3} \delta_{i j} \varepsilon}_{\varepsilon_{i j}: \text { dissipation }}+\underbrace{\phi_{i j}}_{\text {pressure-strain }}+\underbrace{S_{R, i j}}_{\text {interfacial }}}_{P_{1}\left(\overline{u_{i}^{\prime} u_{k}^{\prime}} \frac{\partial \overline{u_{j}}}{\partial x_{k}}+\overline{u_{j}^{\prime} u_{k}^{\prime}} \frac{\partial \overline{u_{i}}}{\partial x_{k}}\right)}
\end{aligned}
$$

with

$$
\begin{aligned}
& S_{R, i j}=b_{i j}^{*} S_{k} \\
& =\left(\begin{array}{ccc}
\underbrace{\min \left(0.67+0.67 \exp \left(370 R e_{\mathrm{p}}^{-1.2}\right), 2\right)}_{b_{11}^{*}} & 0 & 0 \\
0 & \underbrace{\frac{1}{2}\left(2-b_{11}^{*}\right)}_{b_{22}^{*}} & 0 \\
0 & 0 & b_{33}^{*}=b_{22}^{*}
\end{array}\right) S_{k}
\end{aligned}
$$


where $S_{R, i j}$ and $S_{k}$ denote the BIT sources for Reynolds stresses and turbulent kinetic energy, respectively. Here, $S_{k}$ is adopted from Ma et al. (2017), which has been validated for a number of bubbly flow cases (Liao et al., 2019).

$$
S_{k}=\min \left(0.18 \cdot R e_{\mathrm{p}}^{0.23}, 1\right) \boldsymbol{F}_{\mathrm{D}} \cdot \boldsymbol{u}_{\mathrm{r}}
$$

The modelled pressure-strain term $\phi_{j}$ in Eq. (2) is identical to the SSG model (Speziale et al., 1991), and its two-phase version is given in Appendix A1. $\alpha_{1}$ is the liquid volume fraction, $v_{1}$ is the liquid molecular kinematic viscosity, and $c_{\mathrm{s}}=1.63$ is an empirical constant. Moreover, the bubble Reynolds number $R e_{\mathrm{p}}=d_{\mathrm{p}} u_{\mathrm{r}} / v$ is based on bubble diameter $d_{\mathrm{p}}, \boldsymbol{u}_{\mathrm{r}}$ is the mean relative velocity between the liquid and bubble (with $u_{\mathrm{r}} \equiv\left\|\boldsymbol{u}_{\mathrm{r}}\right\|$ ), and the drag force reads $\boldsymbol{F}_{\mathrm{D}}=\frac{3}{4 d_{\mathrm{p}}} C_{\mathrm{D}} \alpha_{\mathrm{g}}\left\|\boldsymbol{u}_{\mathrm{r}}\right\| \boldsymbol{u}_{\mathrm{r}}$.

The rate of turbulence dissipation $\varepsilon$ in Eq. (2) can be obtained from its own transport equation:

$$
\begin{aligned}
\frac{\mathrm{D}\left(\alpha_{1} \varepsilon\right)}{\mathrm{D} t}= & \alpha_{1} \underbrace{{C_{\varepsilon 1}}_{\varepsilon_{k}} \frac{\varepsilon}{k}}_{P_{\varepsilon}}+\underbrace{\frac{\partial}{\partial x_{k}}\left[\alpha_{1}\left(v_{1}+\frac{v_{\mathrm{t}}}{\sigma_{\varepsilon}}\right) \frac{\partial \varepsilon}{\partial x_{k}}\right]}_{D_{\varepsilon}} \\
& -\underbrace{\alpha_{1} C_{\varepsilon 2} \frac{\varepsilon^{2}}{k}}_{\varepsilon_{\varepsilon}}+\underbrace{0.3 C_{\mathrm{D}} \frac{S_{k}}{\tau}}_{S_{\varepsilon}}
\end{aligned}
$$

with all single-phase related terms having the standard form (Hanjalic and Launder, 2011), and the empirical constants are $C_{\varepsilon 1}=1.45, \sigma_{\varepsilon}=1.36$, and $C_{\varepsilon 2}=1.83$. The interfacial term for the $\varepsilon$-equation, $S_{\varepsilon}$, was modelled in $\mathrm{Ma}$ et al. (2017) and is adopted here without modification, i.e., $S_{\varepsilon}=0.3 C_{\mathrm{D}} S_{k} / \tau$. In this expression, $S_{k}$ is given by Eq. (4), $C_{D}$ is the drag coefficient, and

$$
\tau=\frac{d_{\mathrm{p}}}{u_{\mathrm{r}}}
$$

is the time scale characterizing BIT. The major advancement in this model is that not only the source terms $S_{k}$ and $S_{\varepsilon}$, but also the expression of $b_{i j}^{*}$ in Eq. (3) are derived on the basis of the DNS data from Santarelli and Fröhlich $(2015,2016)$

\section{Investigated cases and numerical settings}

The Reynolds-stress model presented above is applied in simulating the vertical pipe flows of Hosokawa and Tomiyama (2013), where the unladen bulk flow is laminar in the experiment and a homogeneous bubble column case of Akbar et al. (2012). These cases are chosen in order to minimize the effect of SIT as well as its interaction with BIT, which is desirable for a pure validation of BIT closures. Furthermore, the dataset provides full Reynolds-stress components of the liquid phase, which is rare in the published experimental data. Besides the comprehensive turbulence information in the carrier phase, the dispersed phase in the presently considered cases exhibits constant bubble diameters, leading to another benefit for validating BIT model, namely, we can focus on checking the RSM without being influenced by the uncertainty caused by modelling the polydispersity in the momentum and turbulence equations.

The experimental conditions of the three pipe flow cases are briefly summarized in Table 1 . The bulk Reynolds number $R e_{\mathrm{b}}=900$ is defined according to the bulk liquid velocity $J_{1}$ and pipe diameter $D$, which is the same for all the cases considered here. Water and air were fed into a vertical pipe of $2 \mathrm{~m}$ in height at room temperature and atmospheric pressure (see Fig. 1). The gravitational force acts in the negative $x$-direction. Gas volume fraction, mean liquid/gas velocity, and Reynolds-stresses were measured radially at an axial distance of $1.7 \mathrm{~m}$ from the bottom, where the flow is regarded fully developed. The complete experimental setup and the applied measurement techniques are described in Hosokawa and Tomiyama (2013), and we, therefore, refer the reader to that paper for more details.

The homogeneous bubble column case of Akbar et al. (2012) is used as another validation case. This case features a rectangular water/air bubble column, with a gas superficial velocity of $3 \mathrm{~mm} / \mathrm{s}$. Its height $(x)$, width $(y)$, and depth $(z)$ are 800,240 , and $72 \mathrm{~mm}$, respectively. Here, the coordinate system of the previous cases is used for improved readability, which is different from the one employed in the original paper. The global gas void fraction is $1.285 \%$, and the bubble

Table 1 Experimental conditions for pipe flow cases

\begin{tabular}{ccccccc}
\hline $\begin{array}{c}\text { Case } \\
\text { No. }\end{array}$ & $R e_{\mathrm{b}}$ & $\begin{array}{c}\text { Pipe } \\
\text { diameter, } \\
D(\mathrm{~mm})\end{array}$ & $J_{1}(\mathrm{~m} / \mathrm{s})$ & $J_{\mathrm{g}}(\mathrm{m} / \mathrm{s})$ & $\begin{array}{c}\text { Bubble } \\
\text { diameter, } \\
d_{\mathrm{p}}(\mathrm{mm})\end{array}$ & $\begin{array}{c}\text { Void } \\
\text { fraction, } \\
\alpha_{\mathrm{g}}(\%)\end{array}$ \\
\hline $\mathrm{H} 1$ & & 20 & $4.5 \times 10^{-2}$ & $0.4 \times 10^{-3}$ & 3.48 & 0.18 \\
$\mathrm{H} 2$ & 900 & 20 & $4.5 \times 10^{-2}$ & $1.6 \times 10^{-3}$ & 3.52 & 0.71 \\
$\mathrm{H} 3$ & & 20 & $4.5 \times 10^{-2}$ & $3.6 \times 10^{-3}$ & 3.59 & 1.56 \\
\hline
\end{tabular}

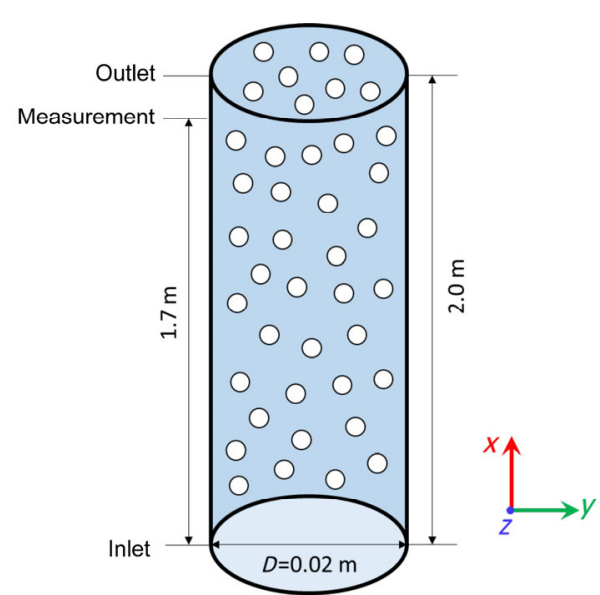

Fig. 1 Sketch of the vertical test section and measurement position. 
size distributions are very close to monodisperse with $d_{\mathrm{p}}=4.37 \mathrm{~mm}$ (Akbar et al., 2012). The data for comparison are taken along the measurement line from the wall to the centre (half the column width) at a height of $500 \mathrm{~mm}$ in the midplane $(z=36 \mathrm{~mm})$, see Fig. 2. More details are provided in the cited reference.

The simulations in the present study are performed with the ANSYS CFX 19.5 software. The computational domain and setup are similar to those adopted in our previous work (Liao et al., 2020), where the same pipe flow cases were investigated using OpenFOAM 6 with an SST $k-\varepsilon$ turbulence model including the BIT term of Ma et al. (2017). Interphase momentum transfer resulting from both drag and non-drag forces, i.e., shear and wall lift, turbulence dispersion, and virtual mass, is taken into account. The corresponding interfacial transfer models employed here are summarized in Table 2. A complete description and further validation studies about all interfacial transfer models can be found in the so-called baseline model (Liao et al., 2015, 2018; Rzehak et al., 2017; Ziegenhein et al. 2017; Colombo et al., 2021)

It is important to stress that our previous study (Liao et al., 2020), however, could not well reproduce the radial void fraction distribution in the pipe flow cases (shown in Section 4). The reason is related to the Tomiyama lift model,

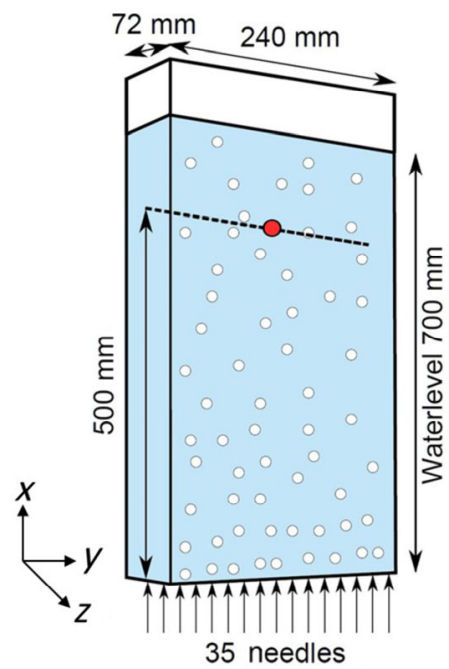

Fig. 2 Sketch of the bubble column of Akbar et al. (2012) and measurement position.

Table 2 Summary of bubble force correlations suggested by the baseline model (Liao et al., 2019)

\begin{tabular}{cc}
\hline Force & Reference \\
\hline Drag & Ishii and Zuber (1979) \\
\hline Shear lift & Tomiyama et al. (2002) \\
\hline Wall lift & Hosokawa et al. (2002) \\
\hline Turbulent dispersion & Burns et al. (2004) \\
\hline Virtual mass & Constant coeffcient $C_{\mathrm{VM}}=0.5$ \\
\hline
\end{tabular}

which would cause a wall-peak or intermediate profile of void fraction based on the relatively small bubble sizes in the considered cases. In contrast, core-peak profiles were measured in the experiment for these three cases. Recall that the major objective of the current work is to better understand BIT and validate the Reynolds-stress closure model. If, however, the simulated void fraction does not fit the experimental value, the BIT model cannot be assessed. Hence, a prerequisite is that the uncertainty created by the other submodels is removed as much as possible. To accomplish this, adjustment is performed on the lift coefficient $C_{\mathrm{L}}$ in order to obtain a satisfactory agreement between the simulated and measured void fraction profiles. More specifically, an iterative process was carried out by running EE-RSM simulations simultaneously optimizing the lift coefficient $C_{\mathrm{L}}$, while the other interfacial force models were employed as they are in Table 2 . The adjusted values of $C_{\mathrm{L}}$ for each case are given in Appendix A2.

Considering that the pipe flow is uniform and axisymmetric, a two-degree wedge instead of the whole pipe is simulated, and a quasi-two-dimensional grid with one layer of cells in the circumferential $(z)$ direction is applied. The mesh resolution in the radial and axial directions is presented in Table 3. The maximum cell size is determined based on the study of Liao et al. (2020), which showed a resolution finer than $0.5 \mathrm{~mm}$ and $10 \mathrm{~mm}$ in the radial and axial directions, respectively, is sufficient to provide mesh-independent results (see a study of grid refinement in Appendix A3). For the bubble column case, the full water region $(x<700 \mathrm{~mm})$ is considered with a $175 \times 240 \times 36$ grid in the $x, y$, and $z$ directions, respectively.

Towards boundary conditions, velocity inlet with measured gas/liquid mass flow rates, pressure outlet for pipe flow, and degassing for bubble column is employed. On the walls of all the considered cases, non-slip and free-slip conditions are assumed for the liquid and gas phases, respectively, and the scalable wall function is selected for modelling flow near the wall. A maximum domain imbalance of mass and momentum of $0.1 \%$ and residual of $10^{-5}$ are set as convergence criteria.

Table 3 Mesh and cell information

\begin{tabular}{cccc}
\hline Direction & Number of cells & $\begin{array}{c}\text { Minimum cell } \\
\text { size }(\mathrm{mm})\end{array}$ & $\begin{array}{c}\text { Maximum cell } \\
\text { size }(\mathrm{mm})\end{array}$ \\
\hline Radial & 60 & 0.025 & 0.48 \\
\hline Axial & 800 & 0.5 & 6.52 \\
\hline
\end{tabular}

\section{Numerical results}

\subsection{Vertical pipe flow}

The calculated radial profiles of void fraction, liquid/gas 
velocity, turbulent kinetic energy, and Reynolds stresses using the present RSM have now been validated against the measurements from Hosokawa and Tomiyama (2013). For comparison reason, additional simulations are carried out using other standard models from the literature under exactly the same conditions, namely employing the same overall optimisation procedure described in Section 3. These models are from Cokljat et al. (2006) and Colombo and Fairweather (2015). The former proposed an isotropic BIT source term, i.e., $b_{11}^{*}=b_{22}^{*}=b_{33}^{*}=2 / 3$, and the latter one suggested $b_{11}^{*}=2 b_{22}^{*}=2 b_{33}^{*}=1$, labelled as "1:0.5:0.5" in the following figures. Both models have $b_{i j}^{*}=0$, when $i \neq j$, just the same as the present RSM.

Figure 3 shows the distribution of gas volume fraction. As mentioned above, the profiles are optimized by adjusting the lift coefficient in order to put the focus only on the BIT issue. Consequently, the simulation results, which are illustrated with dotted (for isotropic BIT source), dashed (for "1:0.5:0.5"), and solid (for the present RSM) lines, coincide with each other and agree well with the experimental data in all the three cases. As additional information, the results of our previous work (Liao et al., 2020) employed with the lift coefficient of Tomiyama et al. (2002) are included (see the dash-dot lines). One can notice that Cases H1, H2, and $\mathrm{H} 3$ all exhibit a core-peak profile in the experiment, and the peak increases with the average void fraction and bubble diameter (see Table 1). Based on the lift correlation of Tomiyama et al. (2002), all the cases have a positive lift coefficient $C_{\mathrm{L}}=0.288$ for the considered bubble parameters, resulting a flat void fraction profile in our previous work.

Figure 4 presents the vertical component of gas velocity. As one can see, slight over-prediction exists in all three cases. Nevertheless, the profiles are in consistent with those of void fractions, and the general slope in Cases $\mathrm{H} 1-\mathrm{H} 3$ becomes gradually steeper. Note that the deviation in the near-wall region can be ignored, since the void fraction there is nearly zero. The present three sets of simulations using different values of $b_{11}^{*}, b_{22}^{*}, b_{33}^{*}$ conform well with each other.

Figure 5 shows the vertical component of liquid velocity. The results of all three cases are in good agreement with the experimental data. The peak of the parabolic profiles increases from Cases $\mathrm{H} 1$ to $\mathrm{H} 3$, which corresponds to the void fraction distribution presented in Fig. 3. Even though a small discrepancy exists in the pipe center region in Cases H1, $\mathrm{H} 2$, and $\mathrm{H} 3$, the three simulations with variable distribution of BIT sources are in accordance with each other. It is important to emphasize that the consistency in the prediction of gas volume fraction and velocity profiles shown in Figs. 3-5 is the prerequisite for a fair comparison with the different $b_{i j}^{*}$ approaches in RSM.
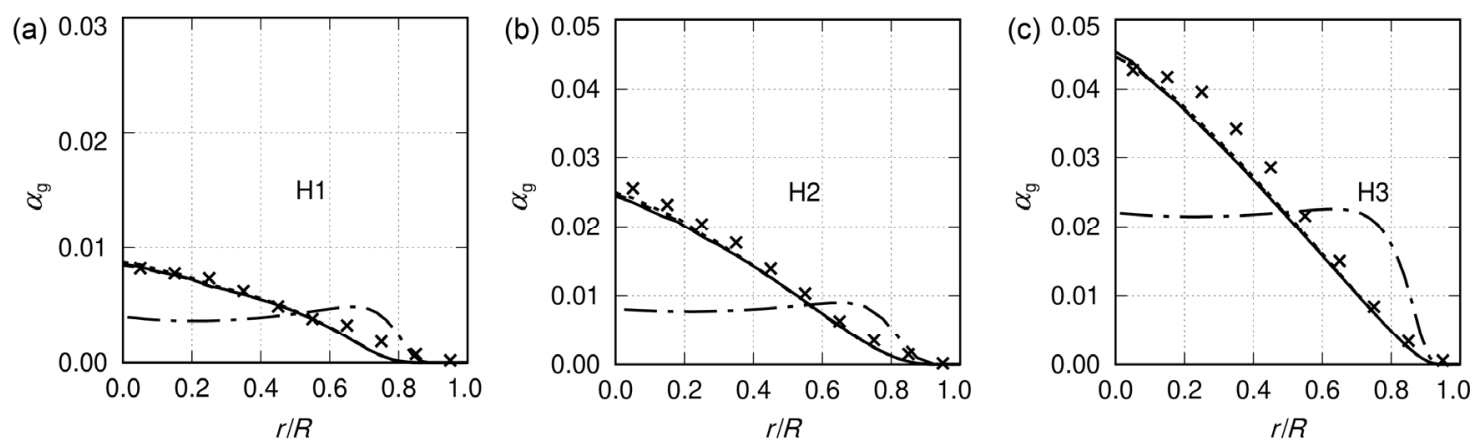

Fig. 3 Radial profile of gas volume fraction (crosses: experimental data from Hosokawa and Tomiyama (2013), dotted line: isotropic BIT source in $x, y, z$ directions, dashed line: BIT source in $x, y, z$ directions is 1:0.5:0.5, solid line: present work, dash-dot line: results from Liao et al. (2020)).
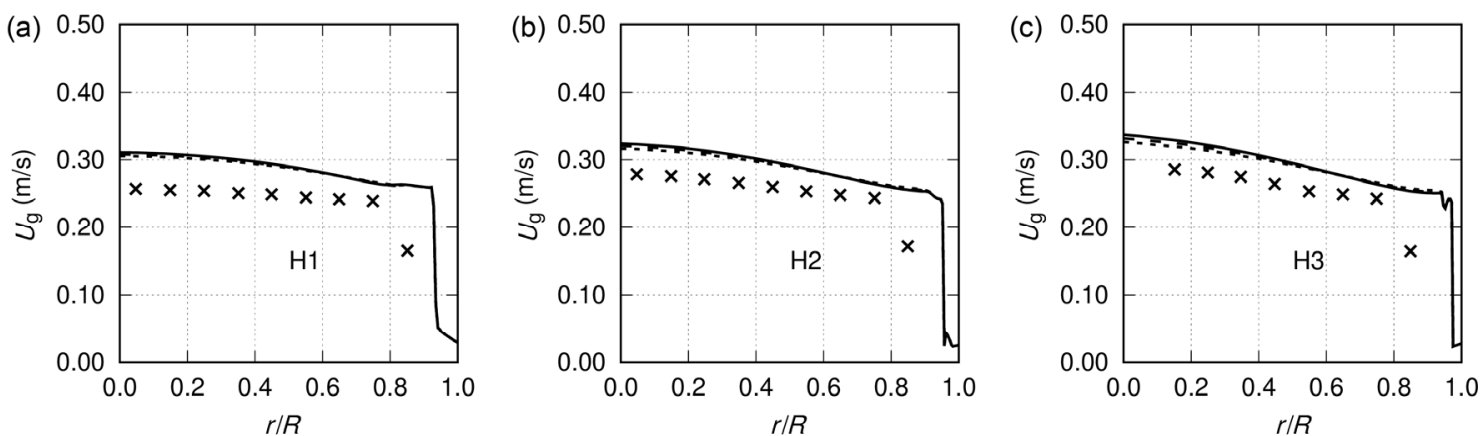

Fig. 4 Radial profile of gas vertical velocity (crosses: experimental data from Hosokawa and Tomiyama (2013), dotted line: isotropic BIT source, dashed line: BIT source in $x, y, z$ directions is 1: 0.5: 0.5 , solid line: present work). 
We now turn to look at the turbulence parameters. As expected, the TKE provided by the three simulations is identical (see Fig. 6), since we keep the source terms for $k$ in Eq. (4) and $\varepsilon$ (involved in Eq. (5)) exactly the same (Ma et al., 2017). In all the core-peak cases, the simulation results agree satisfactorily with the experimental data. It shows that the proposed model for bubble-induced production and destruction of TKE in the liquid phase is reliable.

As aforementioned, the present three sets of simulations only differ in partitioning $k$ source into three components of Reynolds normal stresses, i.e., equally, or with fractions of either 1:0.5:0.5 or $b_{i j}^{*}$ suggested by Eq. (3) in the present work. In the experiment, $\overline{u^{\prime} u^{\prime}}$ was measured approximately twice as large as the other two directions in all these cases over a large range of radial direction (Hosokawa and Tomiyama, 2013), since it is in the slip velocity direction, which contributes to the main BIT source. This component is well reproduced in Fig. 7 by the present work, having the highest value of $\overline{u^{\prime} u^{\prime}}$. The bubble Reynolds numbers in these three pipe cases are very similar $\sim 900$, resulting $b_{11}^{*} \approx 1.4$ in Eq. (3), which is higher than the values based on the "isotropic" and the "1:0.5:0.5" approaches. As expected, the "isotropic" assumption underpredicts $\overline{u^{\prime} u^{\prime}}$ substantially, and the result obtained by "1:0.5:0.5" is in the middle. Additionally, we show in Appendix A4 a simulation without any BIT sources $\left(S_{R, i j}=0\right.$ and $\left.S_{\varepsilon}=0\right)$. The results reveal clearly the importance of adding the BIT terms for calculating Reynolds stresses.

To illustrate the turbulence contributed by different sources in Eq. (2) for the streamwise direction, we plot in Fig. 8 the shear-induced production term $P_{11}$ and the interfacial term $S_{R, 11}$. Although they are terms based on the
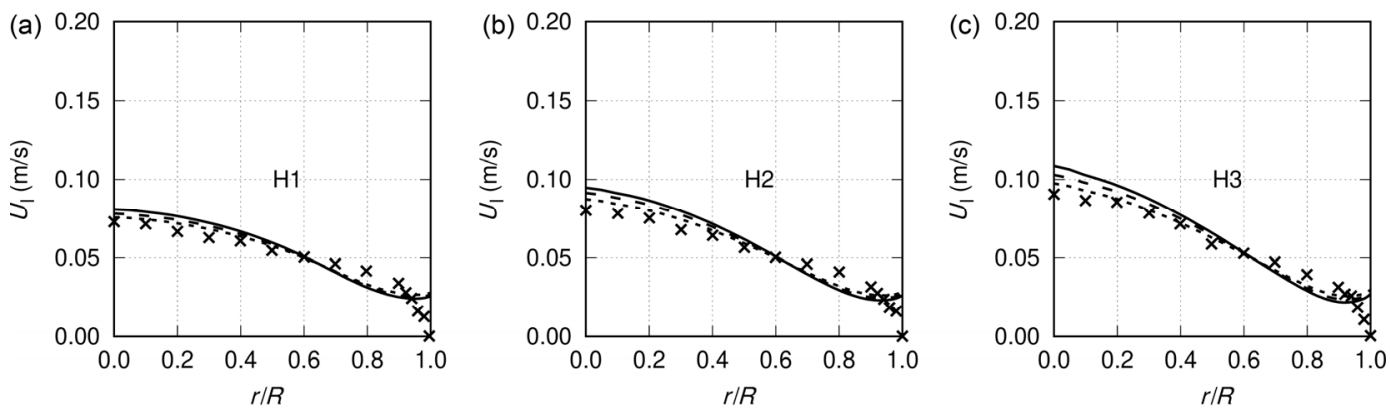

Fig. 5 Radial profile of liquid vertical velocity (crosses: experimental data from Hosokawa and Tomiyama (2013), dotted line: isotropic BIT source in $x, y, z$ directions, dashed line: BIT source in $x, y, z$ directions is 1:0.5:0.5, solid line: present work).
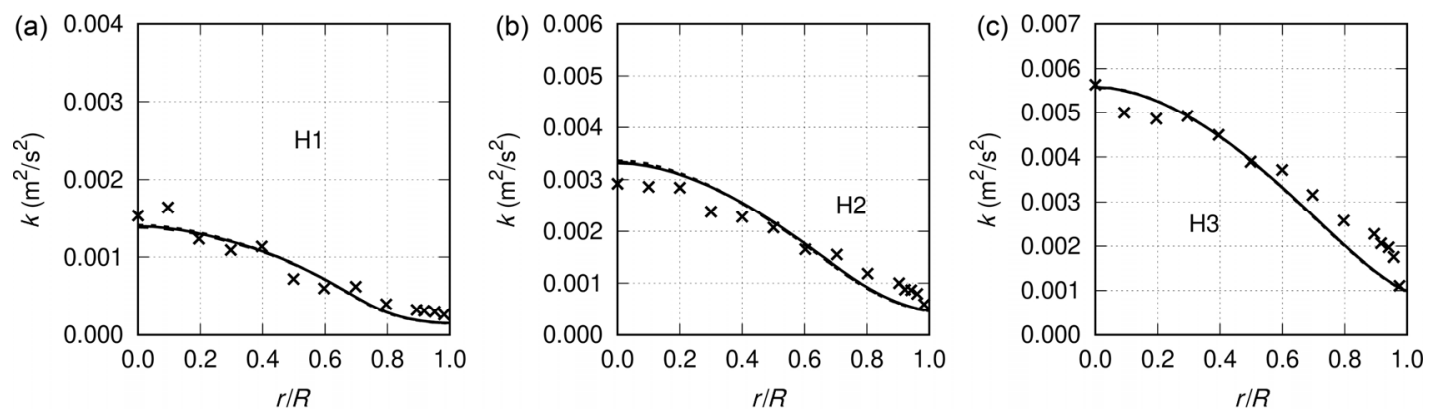

Fig. 6 Radial profile of turbulent kinetic energy (crosses: experimental data from Hosokawa and Tomiyama (2013), dotted line: isotropic BIT source in $x, y, z$ directions, dashed line: BIT source in $x, y, z$ directions is 1:0.5:0.5, solid line: present work).
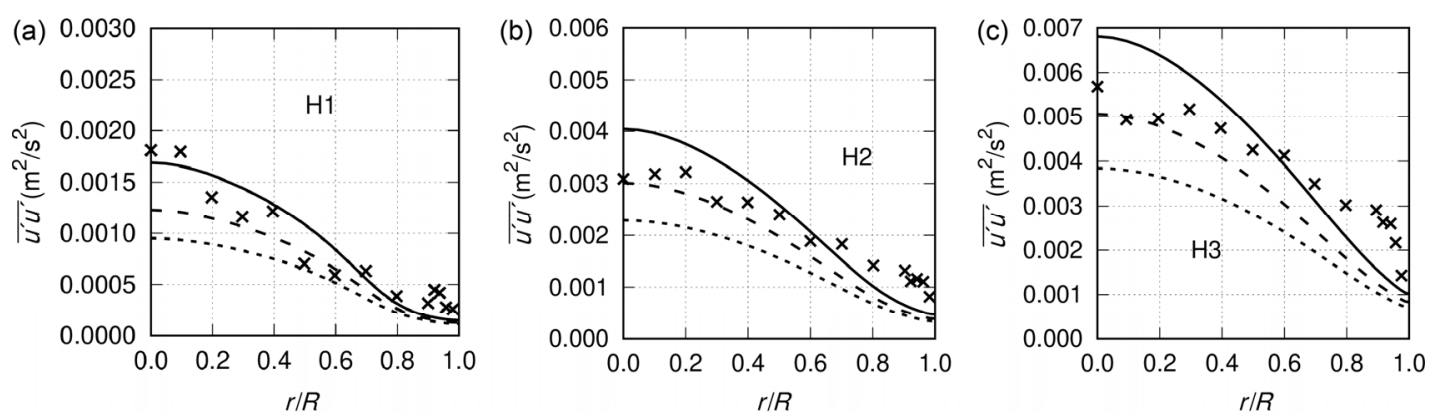

Fig. 7 Radial profile of Reynolds stress $\overline{u^{\prime} u^{\prime}}$ (crosses: experimental data from Hosokawa and Tomiyama (2013), dotted line: isotropic BIT source in $x, y, z$ directions, dashed line: BIT source in $x, y, z$ directions is 1:0.5:0.5, solid line: present work). 
RSM modelling equation, it indicates an interesting issue in the present flow configuration. The unladen background flow is indeed laminar in the experiment (Hosokawa and Tomiyama, 2013). By adding bubbles, the flow, however, becomes turbulent and initializes the same time as well the shear-induced contribution if there is a mean flow gradient (in the case for all the considered cases here). The shearinduced contribution compared to the interfacial term is generally small in the present BIT-dominated flows over a wide range across the pipe, but the contribution is not anywhere negligible (e.g., close to the wall where the shear rate is relatively high).

Figures 9 and 10, depicting Reynolds normal stresses in the $y$ and $z$ directions, are measured approximately equal in the experiment. This is due to the fact that in the BITdominated cases, the turbulence is generated by the bubble wakes, which individually can be approximated by the axisymmetric turbulence framework to some extent (George and Hussein, 1991; Ma et al., 2020b). Again, the present work gives generally superior results to the other two approaches, which obviously predict significantly higher levels of both components.

We plot in Fig. 11 the single non-zero Reynolds shear stress component $\overline{u^{\prime} v^{\prime}}$. In contrast to the components of Reynolds normal stresses, the different BIT source terms surprisingly produce the same $\overline{u^{\prime} v^{\prime}}$ in all the considered cases. The reason for this issue relates to $b_{i j}^{*}=0$ for $i \neq j$, so that in Eq. (3) there is no direct BIT source for this component. Here, the real source term in the transport equation of $\overline{u^{\prime} v^{\prime}}$ is the pressure-strain term $\phi_{12}$ in Eq. (2), which is less sensitive by comparing to define a direct BIT source. It is encouraging to see that the calculated Reynolds shear stresses are relatively close to the experimental data. In contrast, a large deviation was observed in our previous
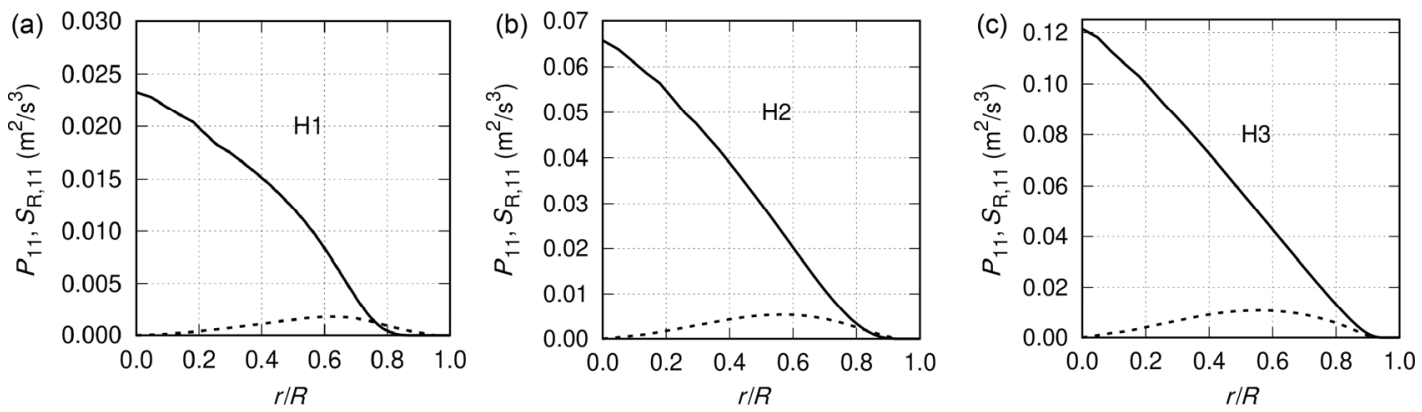

Fig. 8 Production and interfacial source terms for normal Reynolds stresses (solid line: $S_{R, 11}$, dotted line: $P_{11}$ ).
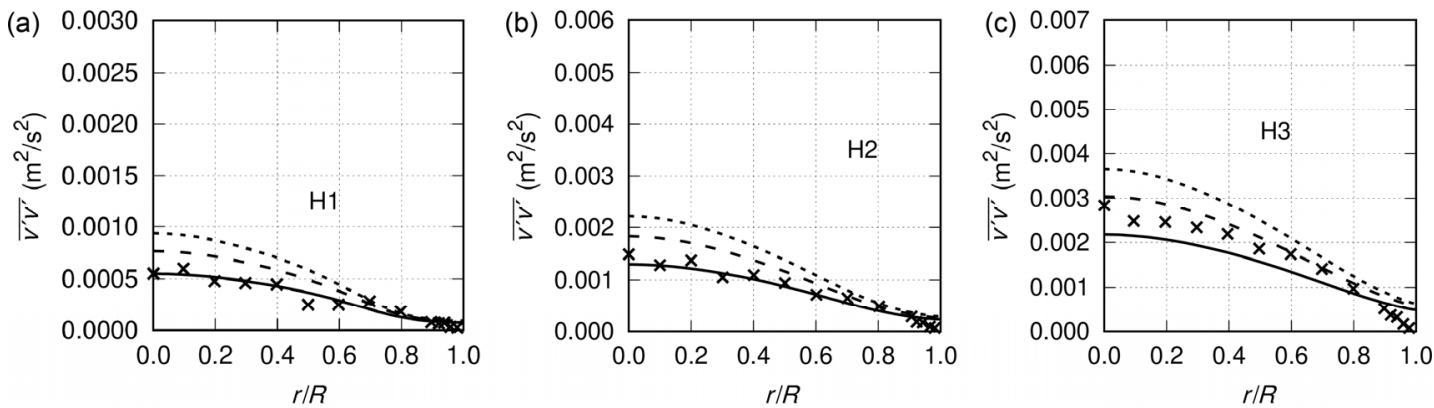

Fig. 9 Radial profile of Reynolds stress $\overline{v^{\prime} v^{\prime}}$ (crosses: experimental data from Hosokawa and Tomiyama (2013), dotted line: isotropic BIT source in $x, y, z$ directions, dashed line: BIT source in $x, y, z$ directions is 1:0.5:0.5, solid line: present work).
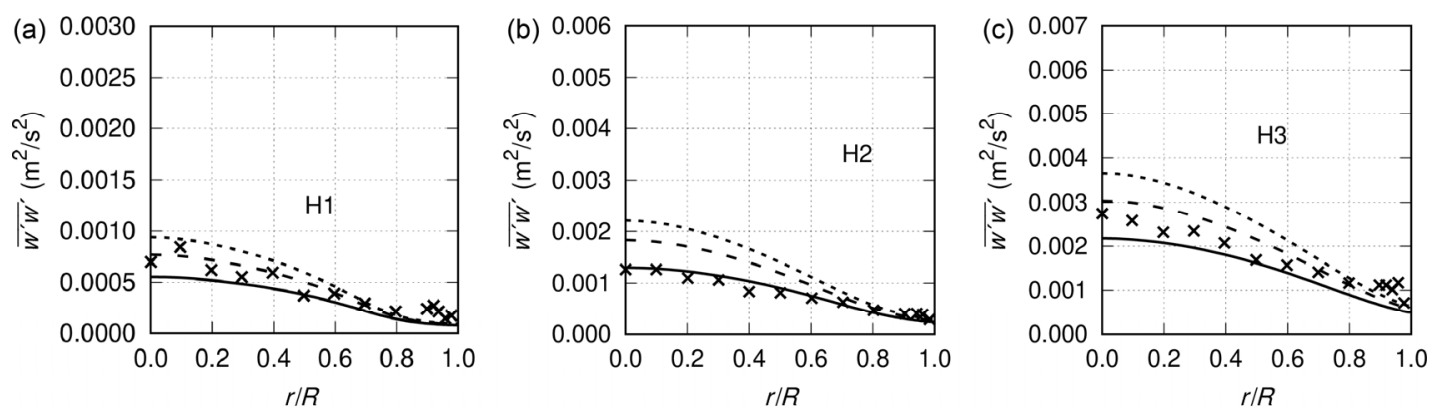

Fig. 10 Radial profile of Reynolds stress $\overline{w^{\prime} w^{\prime}}$ (crosses: experimental data from Hosokawa and Tomiyama (2013), dotted line: isotropic BIT source in $x, y, z$ directions, dashed line: BIT source in $x, y, z$ directions is 1:0.5:0.5, solid line: present work). 
work (Liao et al., 2020) using the Boussinesq hypothesis in the framework of a two-equation eddy-viscosity turbulence model.

\subsection{A bubble column case}

While in the previous subsection we have tested the RSMs for bubbly flow in a pipe with varying gas void fraction but a similar bubble Reynolds number, we consider in the following a bubble column case (Akbar et al., 2012) with different bubble sizes and without bulk velocity.

Three EE-RSM simulations based on different BIT sources were performed for this case. Based on own previous work, the same set of interfacial force models as detailed by Liao et al. (2019) (Table 2) were employed to achieve good agreement of gas void fraction and vertical liquid/gas velocity at all simulations (see Fig. 12). The flatter profiles for the liquid and gas velocities may be due to the slight inaccuracy in the gas void fraction close to the wall. Concerning the liquid Reynolds stresses, as shown in Fig. 13, the present BIT model provides again the most accurate results among these three BIT models, as well as our own previous work using large eddy simulation (Ma et al., 2015b). The ratio of the Reynolds normal stresses $\overline{u^{\prime} u^{\prime}}: \overline{v^{\prime} v^{\prime}} \approx 2.5: 1$ observed in the experiment is very well reproduced for the centre region of the measurement line.

\section{Conclusions}

In the present work, a full Reynolds-stress model recently proposed by Ma et al. (2020b) based on the budget analysis of the Reynolds stress and its dispersion from DNS for
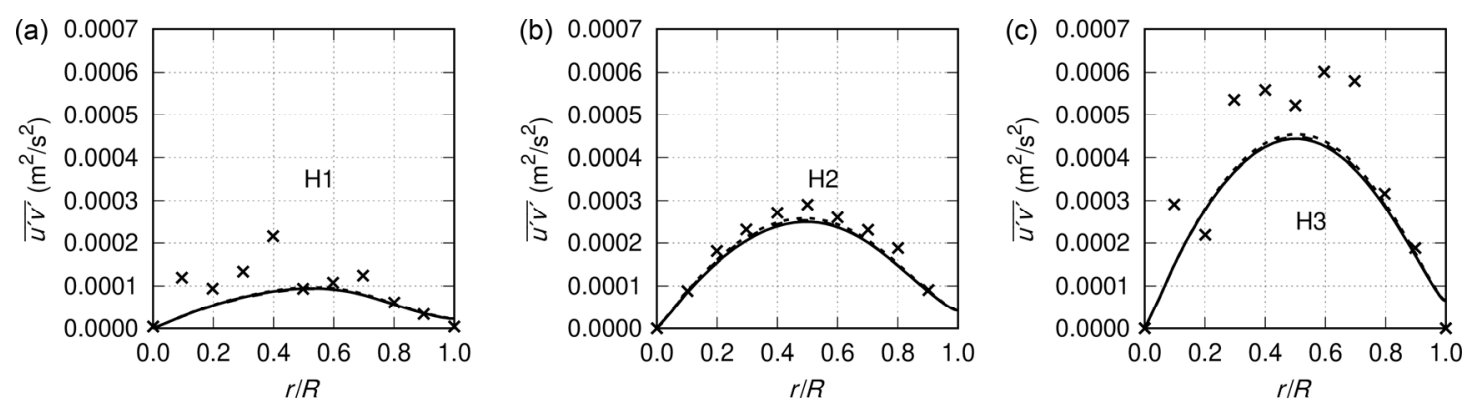

Fig. 11 Radial profile of Reynolds stress $\overline{u^{\prime} v^{\prime}}$ (crosses: experimental data from Hosokawa and Tomiyama (2013), dotted line: isotropic BIT source in $x, y, z$ directions, dashed line: BIT source in $x, y, z$ directions is 1:0.5:0.5, solid line: present work).
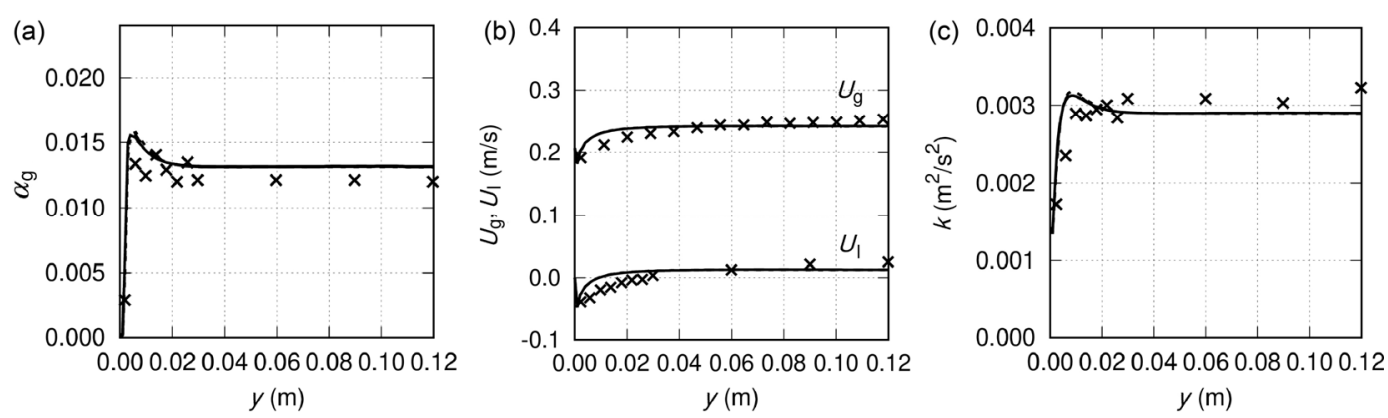

Fig. 12 Comparison of numerical results with experimental data of Akbar et al. (2012): (a) gas void fraction, (b) liquid vertical velocity and gas vertical velocity, and (c) liquid TKE (crosses: experimental data, dotted line: isotropic BIT source in $x, y$, $z$ directions, dashed line: BIT source in $x, y, z$ directions is 1:0.5:0.5, solid line: present work).
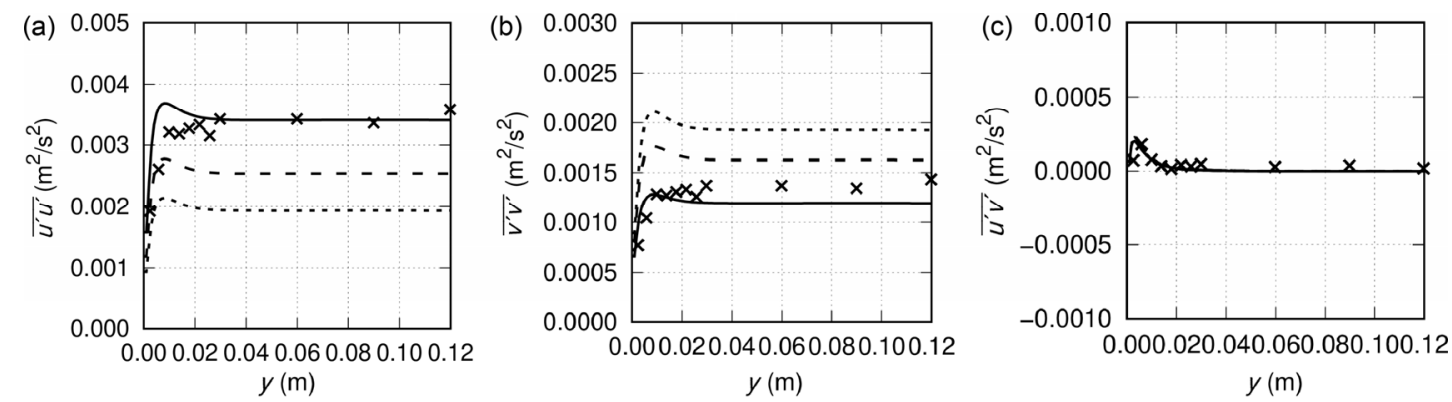

Fig. 13 Comparison of calculated and measured Reynolds-stress components (crosses: experimental data of Akbar et al. (2012), dotted line: isotropic BIT source in $x, y, z$ directions, dashed line: BIT source in $x, y, z$ directions is 1:0.5:0.5, solid line: present work). 
bubbly flows is tested rigorously for a large number of bubbly flows. The results of other two standard models (labelled as "isotropic" and "1:0.5:0.5", respectively) are included in the comparison.

In all EE-RSM simulations, the lift force coefficient has been carefully fine tuned, so that the simulated gas void fraction fits very well to its experimental distributions. This procedure removes the uncertainty created by the other submodels as much as possible and provides an optimal condition for the comparison of different BIT turbulence models. As expected, the model proposed by $\mathrm{Ma}$ et al. (2020b) leads to a substantial improvement for all the considered cases with or without bulk flow involving different void fractions and bubble Reynolds numbers and should be the RSM of choice for dispersed bubbly flow applications.

The present BIT model is now tested for steady fully developed bubbly flows with very encouraging results. In the future work, we will implement this BIT model within the scale-resolving simulations framework (Fröhlich and von Terzi, 2008) as suggested by Ullrich et al. (2021), so that the Euler-Euler approach coupled with steady or unsteady RANS modelling can accurately predict the turbulence parameters both for the steady full developed cases, as well as unsteady cases, like bubble plume.

\section{Appendix}

\section{A1 Pressure-strain term in the present study (Speziale et al., 1991)}

The modelled pressure-strain term $\phi_{i j}$ can be expressed in terms of the Reynolds-stress anisotropy tensor $a_{i j}$, its second invariant $A_{2}$, stress production tensor $P_{i j}$, its complement $E_{i j}$, and mean rate of strain $F_{i j}$ :

$$
\begin{aligned}
& \phi_{i j}=-c_{1} \alpha_{1} \varepsilon a_{i j}+c_{1}^{\prime} \alpha_{1} \varepsilon\left(a_{i k} a_{k j}-\frac{1}{3} \delta_{i j} A_{2}\right) \\
& -c_{2}^{*}\left(P_{i j}-\frac{1}{3} \delta_{i j} P_{k k}\right)-c_{3}^{*}\left(E_{i j}-\frac{1}{3} \delta_{i j} E_{k k}\right)-c_{4}^{*} \alpha_{1} k F_{i j}-c_{5}^{*} a_{i j} P_{k k}(\mathrm{~A} 1)
\end{aligned}
$$

where

$$
\begin{aligned}
& a_{i j}=\frac{\overline{u_{i}^{\prime} u_{j}^{\prime}}}{k}-\frac{2}{3} \delta_{i j}, \quad A_{2}=a_{j i} a_{i j}, \\
& E_{i j}=-\alpha_{1}\left(\overline{u_{i}^{\prime} u_{k}^{\prime}} \frac{\partial \overline{u_{k}}}{\partial x_{j}}+\overline{u_{j}^{\prime} u_{k}^{\prime}} \frac{\partial \overline{u_{k}}}{\partial x_{i}}\right), \quad F_{i j}=\frac{1}{2}\left(\frac{\partial \overline{u_{i}}}{\partial x_{j}}+\frac{\partial \overline{u_{j}}}{\partial x_{i}}\right)
\end{aligned}
$$

The constants in Eq. (A1) are as follows (Hanjalic and Launder, 2011):

$$
\begin{aligned}
& c_{1}=1.7, \quad c_{1}^{\prime}=1.05, \quad c_{2}^{*}=0.4125, \quad c_{3}^{*}=0.2125, \\
& c_{4}^{*}=0.033+0.65 A_{2}^{1 / 2}, \quad c_{5}^{*}=0.45
\end{aligned}
$$

\section{A2 Adjusted lift coefficient}

The adjusted lift coefficients for the considered cases are listed in Table A1.

Table A1 Adjusted lift coefficients

\begin{tabular}{cccc}
\hline \multirow{2}{*}{ Case No. } & \multicolumn{3}{c}{$C_{\mathrm{L}}$} \\
\cline { 2 - 4 } & Isotropic & $1: 0.5: 0.5$ & Present work \\
\hline H1 & 0.05 & 0.0375 & 0.01 \\
\hline H2 & -0.065 & -0.08 & -0.1 \\
\hline H3 & -0.11 & -0.13 & -0.15 \\
\hline
\end{tabular}

\section{A3 Grid refinement study for Case H3}

We present the results for Case H3 using four different meshes in Fig. A1. All results displayed in the main text are from the second mesh with the grid numbers 41 and 801 in radial and axial directions, respectively.

\section{A4 Case H3 without BIT terms}

In Fig. A2, we present the results of Case H3 without considering BIT terms $\left(S_{R, i j}=0\right.$ and $\left.S_{\varepsilon}=0\right)$.
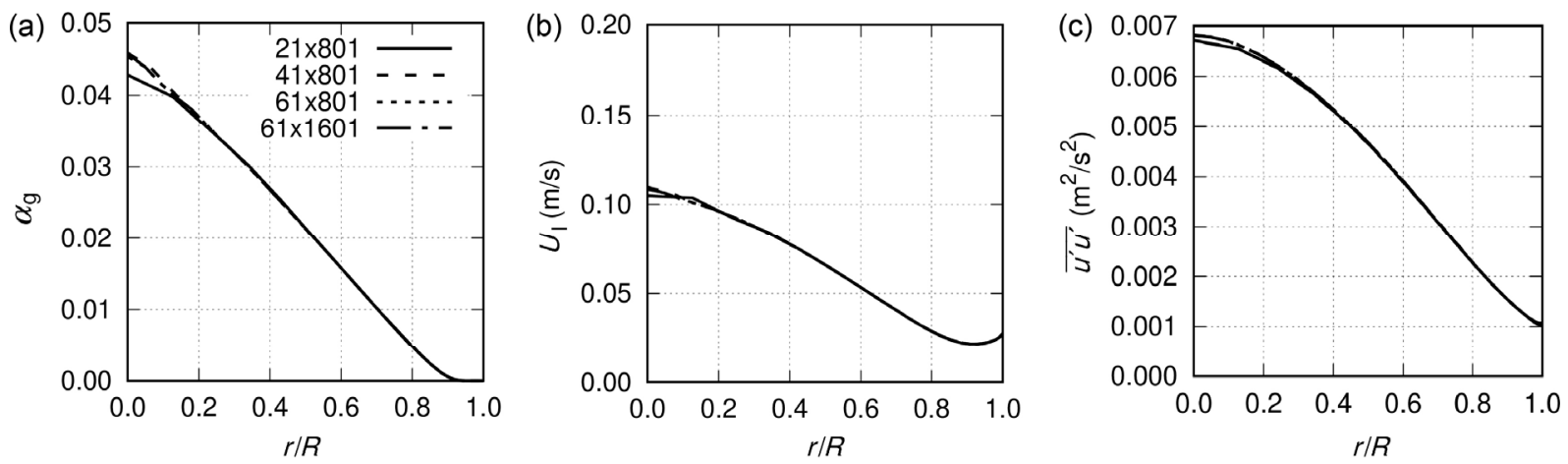

Fig. A1 Comparison of the gas void fraction, mean liquid velocity, and $\overline{u^{\prime} u^{\prime}}$ obtained by four different meshes. 
(a)

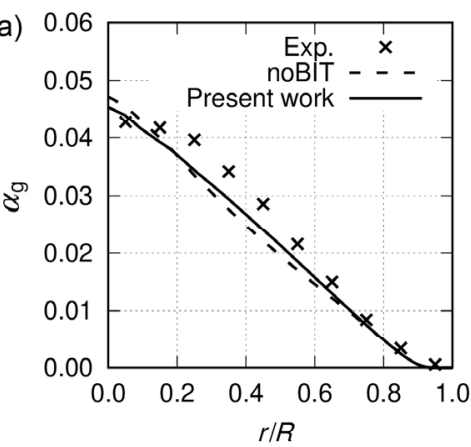

(b)

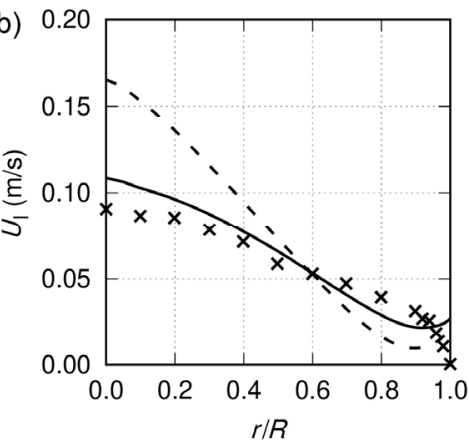

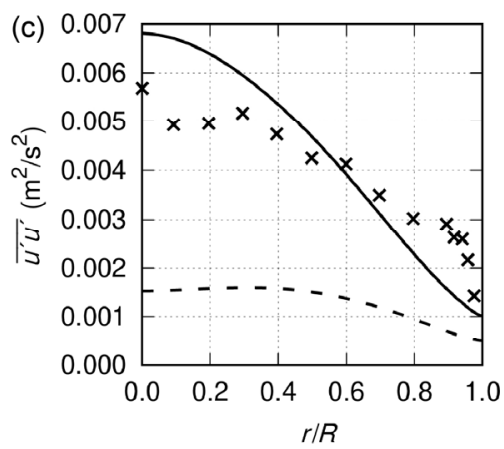

Fig. A2 Comparison of the gas void fraction, mean liquid velocity, and $\overline{u^{\prime} u^{\prime}}$ calculated by using the present BIT terms and without any BIT term.

\section{Acknowledgements}

The authors benefited from some discussions on this topic with Dirk Lucas and Andy Bragg. Tian Ma acknowledges funding by Deutsche Forschungsgemeinschaft (DFG, German Research Foundation) under Grants MA 8408/1-1 and MA 8408/2-1.

\section{Funding note}

Open Access funding enabled and organized by Projekt DEAL.

\section{Declaration of competing interest}

The authors have no competing interests to declare that are relevant to the content of this article.

\section{References}

Akbar, M., Hayashi, K., Hosokawa, S., Tomiyama, A. 2012. Bubble tracking simulation of bubble-induced pseudoturbulence. Multiphase Sci Technol, 24: 197-222.

Balachandar, S., Eaton, J. K. 2010. Turbulent dispersed multiphase flow. Annu Rev Fluid Mech, 42: 111-133.

Biesheuvel, A., van Wijngaarden, L. 1984. Two-phase flow equations for a dilute dispersion of gas bubbles in liquid. J Fluid Mech, 148: 301-318.

Bragg, A. D., Liao, Y., Fröhlich, J., Ma, T. 2021. Assessment of the validity of a log-law for wall-bounded turbulent bubbly flows. Int J Heat Fluid Flow, 91: 108857.

Burns, A. D., Frank, T., Hamill, I., Shi, J. M. 2004. The Favre averaged drag model for turbulent dispersion in Eulerian multi-phase flows. In: Proceedings of the 5th International Conference on Multiphase Flow, Yokohama, Japan, Paper No. 392.

Cokljat, D., Slack, M., Vasquez, S. A., Bakker, A., Montante, G. 2006. Reynolds-stress model for eulerian multiphase. Progr Comput Fluid Dynam Int J, 6: 168-178.

Colombo, M., Fairweather, M. 2015. Multiphase turbulence in bubbly flows: RANS simulations. Int J Multiphase Flow, 77: 222-243.
Colombo, M., Rzehak, R., Fairweather, M., Liao, Y., Lucas, D. 2021. Benchmarking of computational fluid dynamic models for bubbly flows. Nucl Eng Des, 375: 111075.

Deen, N. G., Solberg, T., Hjertager, B. H. 2001. Large eddy simulation of the gas-liquid flow in a square cross-sectioned bubble column. Chem Eng Sci, 56: 6341-6349.

Du Cluzeau, A., Bois, G., Toutant, A. 2019. Analysis and modelling of Reynolds stresses in turbulent bubbly up-flows from direct numerical simulations. J Fluid Mech, 866: 132-168.

Fröhlich, J., von Terzi, D. 2008. Hybrid LES/RANS methods for the simulation of turbulent flows. Prog Aerosp Sci, 44: 349-377.

George, W. K., Hussein, H. J. 1991. Locally axisymmetric turbulence. J Fluid Mech, 233: 1-23.

Hanjalic, K., Launder, B. 2011. Modelling Turbulence in Engineering and the Environment. Cambridge: Cambridge University Press.

Hosokawa, S., Tomiyama, A. 2013. Bubble-induced pseudo turbulence in laminar pipe flows. Int J Heat Fluid Flow, 40: 97-105.

Hosokawa, S., Tomiyama, A., Misaki, S., Hamada, T. 2002. Lateral migration of single bubbles due to the presence of wall. In: Proceedings of the ASME 2002 Joint U.S.-European Fluids Engineering Division Conference, Quebec, Canada.

Ilić, M. 2006. Statistical analysis of liquid phase turbulence based on direct numerical simulations of bubbly flows. Ph.D. Thesis. Forschungszentrum Karlsruhe, Germany.

Ishii, M., Hibiki, T. 2006. Thermo-Fluid Dynamics of Two-Phase Flow. Boston, MA, USA: Springer.

Ishii, M., Zuber, N. 1979. Drag coefficient and relative velocity in bubbly, droplet or particulate flows. AIChE J, 25: 843-855.

Kataoka, I., Serizawa, A. 1989. Basic equations of turbulence in gasliquid two-phase flow. Int J Multiphase Flow, 15: 843-855.

Launder, B. E., Reece, G. J., Rodi, W. 1975. Progress in the development of a Reynolds-stress turbulence closure. J Fluid Mech, 68: 537-566.

Liao, Y. 2020. Update to the MUSIG model in ANSYS CFX for reliable modelling of bubble coalescence and breakup. Appl Math Model, 81: 506-521.

Liao, Y., Lucas, D., Krepper, E. 2014. Application of new closure models for bubble coalescence and breakup to steam-water vertical pipe flow. Nucl Eng Des, 279: 126-136.

Liao, Y., Ma, T., Krepper, E., Lucas, D., Fröhlich, J. 2019. Application of a novel model for bubble-induced turbulence to bubbly flows in containers and vertical pipes. Chem Eng Sci, 202: 55-69. 
Liao, Y., Ma, T., Liu, L., Ziegenhein, T., Krepper, E., Lucas, D. 2018. Eulerian modelling of turbulent bubbly flow based on a baseline closure concept. Nucl Eng Des, 337: 450-459.

Liao, Y., Rzehak, R., Lucas, D., Krepper, E. 2015. Baseline closure model for dispersed bubbly flow: Bubble coalescence and breakup. Chem Eng Sci, 122: 336-349.

Liao, Y., Upadhyay, K., Schlegel, F. 2020. Eulerian-Eulerian two-fluid model for laminar bubbly pipe flows: Validation of the baseline model. Comput Fluids, 202: 104496.

Liu, L., Keplinger, O., Ma, T., Ziegenhein, T., Shevchenko, N., Eckert, S., Yan, H., Lucas, D. 2018. Euler-Euler simulation and X-ray measurement of bubble chain in a shallow container filled with liquid metals. Chem Eng Sci, 192: 288-305.

Lohse, D. 2018. Bubble puzzles: From fundamentals to applications. Phys Rev Fluids, 3: 110504.

Lopez de Bertodano, M., Lahey, R. T., Jones, O. C. 1994. Development of a $k-\varepsilon$ model for bubbly two-phase flow. J Fluids Eng, 116: 128-134.

Lopez de Bertodano, M., Lee, S. J., Lahey, R. T., Drew, D. A. 1990. The prediction of two-phase turbulence and phase distribution phenomena using a Reynolds stress model. J Fluids Eng, 112: 107-113.

Lu, J., Tryggvason, G. 2013. Dynamics of nearly spherical bubbles in a turbulent channel upflow. J Fluid Mech, 732: 166-189.

Lucas, D., Beyer, M., Banowski, M., Seidel, T., Krepper, E., Liao, Y., Apanasevich, P., Gauss, F., Ma, T. 2016. TOPFLOW-Experiments, model development and validation for the qualification of CFD-codes for two-phase flows. Final report. Technical Report. Helmholtz-Zentrum Dresden-Rossendorf eV., Dresden, Germany.

Lucas, D., Krepper, E., Rzehak, R., Liao, Y., Ma, T., Ziegenhein, T. 2015. Status and challenges of CFD-modelling for poly-disperse bubbly flows. In: Proceedings of the 16th International Topical Meeting on Nuclear Reactor Thermal Hydraulics, Chicago, USA.

Ma, T. 2017. A contribution to turbulence modelling in bubbly flows. Ph.D. Thesis. Technische Universität Dresden, Dresden, Germany.

Ma, T., Lucas, D., Bragg, A. D. 2020a. Explicit algebraic relation for calculating reynolds normal stresses in flows dominated by bubble-induced turbulence. Phys Rev Fluids, 5: 084305.

Ma, T., Lucas, D., Jakirlić, S., Fröhlich, J. 2020b. Progress in the second-moment closure for bubbly flow based on direct numerical simulation data. J Fluid Mech, 883, https://doi.org/ $10.1017 /$ jfm.2019.851.

Ma, T., Lucas, D., Ziegenhein, T., Fröhlich, J., Deen, N. G. 2015a. Scale-Adaptive Simulation of a square cross-sectional bubble column. Chem Eng Sci, 131: 101-108.

Ma, T., Ott, B., Fröhlich, J., Bragg, A. D. 2021. Scale-dependent anisotropy, energy transfer and intermittency in bubble-laden turbulent flows. J Fluid Mech, 927: A16.

Ma, T., Santarelli, C., Ziegenhein, T., Lucas, D., Fröhlich, J. 2017. Direct numerical simulation-based Reynolds-averaged closure for bubble-induced turbulence. Phys Rev Fluids, 2: 034301.

Ma, T., Ziegenhein, T., Lucas, D., Krepper, E., Fröhlich, J. 2015b. Euler-Euler large eddy simulations for dispersed turbulent bubbly flows. Int J Heat Fluid Flow, 56: 51-59.

Magolan, B., Lubchenko, N., Baglietto, E. 2019. A quantitative and generalized assessment of bubble-induced turbulence models for gas-liquid systems. Chem Eng Sci: X, 2: 100009.

Masood, R. M. A., Rauh, C., Delgado, A. 2014. CFD simulation of bubble column flows: An explicit algebraic Reynolds stress model approach. Int J Multiphase Flow, 66: 11-25.

Michaelides, E. E., Crowe, C. T., Schwarzkopf, J. D. 2016. Multiphase Flow Handbook, 2nd edn. Boca Raton, FA, USA: CRC Press.

Morel, C. 1997. Turbulence modeling and first numerical simulations in turbulent two-phase flows. Technical Report. CEA/Grenoble, France.

Ničeno, B., Dhotre, M. T., Deen, N. G. 2008. One-equation sub-grid scale (SGS) modelling for Euler-Euler large eddy simulation (EELES) of dispersed bubbly flow. Chem Eng Sci, 63: 3923-3931.

Qi, Y., Mohammad Masuk, A. U., Ni, R. 2020. Towards a model of bubble breakup in turbulence through experimental constraints. Int J Multiphase Flow, 132: 103397.

Riboux, G., Risso, F., Legendre, D. 2010. Experimental characterization of the agitation generated by bubbles rising at high Reynolds number. J Fluid Mech, 643: 509-539.

Rzehak, R., Krepper, E. 2013. Bubble-induced turbulence: Comparison of CFD models. Nucl Eng Des, 258: 57-65.

Rzehak, R., Ziegenhein, T., Kriebitzsch, S., Krepper, E., Lucas, D. 2017. Unified modeling of bubbly flows in pipes, bubble columns, and airlift columns. Chem Eng Sci, 157: 147-158.

Santarelli, C., Fröhlich, J. 2015. Direct numerical simulations of spherical bubbles in vertical turbulent channel flow. Int J Multiphase Flow, 75: 174-193.

Santarelli, C., Fröhlich, J. 2016. Direct numerical simulations of spherical bubbles in vertical turbulent channel flow. Influence of bubble size and bidispersity. Int J Multiphase Flow, 81: 27-45.

Santarelli, C., Roussel, J., Fröhlich, J. 2016. Budget analysis of the turbulent kinetic energy for bubbly flow in a vertical channel. Chem Eng Sci, 141: 46-62.

Sommerfeld, M. 2017. Numerical methods for dispersed multiphase flows. In: Particles in Flows. Advances in Mathematical Fluid Mechanics. Bodnár, T., Galdi, G., Nečasová, Š., Eds. Birkhäuser.

Speziale, C. G., Sarkar, S., Gatski, T. B. 1991. Modelling the pressurestrain correlation of turbulence: An invariant dynamical systems approach. J Fluid Mech, 227: 245-272.

Tomiyama, A., Tamai, H., Zun, I., Hosokawa, S. 2002. Transverse migration of single bubbles in simple shear flows. Chem Eng Sci, 57: 1849-1858.

Troshko, A. A., Hassan, Y. A. 2001. A two-equation turbulence model of turbulent bubbly flows. Int J Multiphase Flow, 27: 1965-2000.

Ullrich, M., Krumbein, B., Maduta, R., Jakirlić, S. 2021. Turbulent flow in a square cross-sectioned bubble column computed by a scale-resolving reynolds-stress model. Chem Eng Sci, 230: 116201.

Vaidheeswaran, A., Hibiki, T. 2017. Bubble-induced turbulence modeling for vertical bubbly flows. Int J Heat Mass Transf, 115: 741-752.

Wörner, M., Erdogan, S. 2013. Toward improved closure relations for the turbulent kinetic energy equation in bubble-driven flows. Chemie Ingenieur Technik, 85: 1131-1136.

Ziegenhein, T., Rzehak, R., Ma, T., Lucas, D. 2017. Towards a unified approach for modelling uniform and non-uniform bubbly flows. Can J Chem Eng, 95: 170-179. 
Open Access This article is licensed under a Creative Commons Attribution 4.0 International License, which permits use, sharing, adaptation, distribution and reproduction in any medium or format, as long as you give appropriate credit to the original author(s) and the source, provide a link to the Creative Commons licence, and indicate if changes were made.

The images or other third party material in this article are included in the article's Creative Commons licence, unless indicated otherwise in a credit line to the material. If material is not included in the article's Creative Commons licence and your intended use is not permitted by statutory regulation or exceeds the permitted use, you will need to obtain permission directly from the copyright holder.

To view a copy of this licence, visit http://creativecommons.org/licenses/by/4.0/. 